\title{
ÉTUDE DES PROPRIÉTÉS ET DE LA QUALITÉ DE CRISTAUX DE CINABRE SYNTHÉTIQUE EN VUE D'APPLICATIONS EN OPTIQUE NON LINÉAIRE $(*)$
}

\author{
P. J. KUPECEK, D. S. CHEMLA et H. LE PERSON \\ Centre National d'Etudes des Télécommunications, \\ 196, rue de Paris, 92220 Bagneux, France
}

(Reçu le 12 novembre 1975, accepté le ler décembre 1975)

\begin{abstract}
Résumé. - Dans cet article nous présentons les résultats d'un ensemble d'études expérimentales sur les propriétés non linéaires optiques de cristaux de cinabre $(\alpha-\mathrm{HgS})$ synthétiques obtenus par croissance hydrothermale. Les mesures des coefficients non linéaires confirment l'intérêt potentiel du matériau. Par contre l'étude critique des qualités optique et cristalline montre que l'utilité pour la réalisation de dispositifs paramétriques des cristaux synthétisés par voie hydrothermale est pour le moment très limitée.

Abstract. - In this communication we present the results of a serie of experimental studies on nonlinear optical properties of cinnabar $(\alpha-\mathrm{HgS})$ crystals, grown by hydrothermal synthesis. The measurement of the nonlinear coefficients confirms the potential interest of the material. Conversely, the critical study of optical and crystalline qualities shows that the usefulness of hydrothermally grown crystals in optical parametric devices is presently limited.
\end{abstract}

1. Introduction. - Le cinabre cristallin $\alpha$-HgS a souvent été présenté comme un matériau prometteur pour la réalisation de dispositifs utilisant ses propriétés optiques non linéaires dans le proche et le moyen infrarouge. Nous présentons dans cette communication un ensemble d'études expérimentales portant sur des échantillons cristallins obtenus par synthèse hydrothermale [1]. Ces études avaient pour objectif de discuter des possibilités effectives d'utilisation de matériaux obtenus par cette méthode de croissance dans des dispositifs optiques paramétriques. Ceci nous a amené dans un premier temps à déterminer les coefficients d'absorption résiduelle et le coefficient non linéaire du second ordre sur le produit de synthèse, confirmant ainsi son intérêt potentiel. Un second ensemble de mesures a eu pour but d'analyser de façon critique les qualités optique et cristalline du matériau obtenu par synthèse hydrothermale. Pour ce faire, nous avons porté notre attention sur l'homogénéité de la transmission et de la génération de second harmonique, sur la régularité de la structure cristalline et sur l'influence éventuelle des macles présentes dans les échantillons étudiés.

L'ensemble des informations obtenues permet alors de discuter de la réalisation de dispositifs paramétriques optiques utilisant du cinabre hydrothermal.

$\left(^{*}\right)$ Ce travail a bénéficié d'une aide financière de la D. G. R. S. T. (contrat $\mathrm{n}^{\circ}$ 71-7-2687).
2. Génération de second harmonique (G.S. H.) à l'accord de phase et qualité optique. - Le cinabre $\alpha-\mathrm{HgS}$ est un matériau cristallin uniaxial, de biréfringence positive $\left(n_{\mathrm{e}}>n_{0}\right)$. Son groupe de symétrie ponctuel étant 32, les relations entre les composantes sur les axes cristallins de la polarisation non linéaire et du champ électrique fondamental s'écrivent :

$$
\begin{aligned}
& P_{x}^{2 \omega}=d_{11}\left(E_{x}^{2}-E_{y}^{2}\right)+2 d_{14} E_{y} E_{z} \\
& P_{y}^{2 \omega}=-2 d_{11} E_{x} E_{y}-2 d_{14} E_{x} E_{z} \\
& P_{z}^{2 \omega}=0 .
\end{aligned}
$$

Lorsque les ondes fondamentale et harmonique sont toutes deux dans le domaine de transparence du cristal, la dispersion est négligeable et les relations deviennent [2] :

$$
\begin{aligned}
P_{x}^{2 \omega} & =d_{11}\left(E_{x}^{2}-E_{y}^{2}\right) \\
P_{y} & =-2 d_{11} E_{x} E_{y} \\
P_{z} & =0 .
\end{aligned}
$$

La G. S. H. à l'accord de phase entre ondes fondamentales de même polarisation est du type $\mathrm{e}+\mathrm{e} \rightarrow \mathrm{o}$ et permet de déterminer la valeur du coefficient non linéaire du second ordre $d_{11}$.

Les indices de réfraction du cinabre ont été mesurés du visible à $11 \mu \mathrm{m}$ par Bond et al. [3]. Les valeurs obtenues peuvent être représentées par deux expressions de type Sellmeier à quatre paramètres et dont l'écart quadratique moyen est inférieur à $1,5 \times 10^{-3}$ [4]. La 
forme générale des expressions adoptées ainsi que les valeurs numériques des coefficients sont réunies dans le tableau I.

\section{TABLeAU I}

Forme générale et coefficients des relations de Sellmeier

$$
\begin{array}{ccccc} 
& \multicolumn{4}{c}{n_{\mathrm{o}, \mathrm{e}}^{2}=A+\frac{B}{\lambda^{2}-C}-D \lambda^{2}} \\
& A & B & C & D \\
n_{\mathrm{o}} & 6,9443 & 0,3665 & 0,1351 & 0,0019 \\
n_{\mathrm{e}} & 8,3917 & 0,5405 & 0,1380 & 0,0027
\end{array}
$$

Pour mesurer le coefficient $d_{11}$ et les angles d'accord de phase, nous avons utilisé trois sources lasers dont les rayonnements se situent dans trois régions différentes du domaine de transparence du cinabre $(0,62 \mu \mathrm{m}$ $15 \mu \mathrm{m})$. Les caractéristiques essentielles de ces sources sont réunies dans le tableau II.

Nous avons utilisé les formules de Sellmeier du tableau I pour déterminer les valeurs des angles d'accord de phase $\theta_{\text {a.c. }}$ pour la G. S. H. en fonction de la longueur d'onde. L'ensemble des valeurs couvre la quasi-totalité du domaine de transparence (Fig. 1). L'écart quadratique moyen de $1,5 \times 10^{-3}$ sur les formules de Sellmeier conduit à une précision de $\pm 1,2^{\circ}$ sur les angles d'accord. Les échantillons de cinabre

\section{TABleau II}

\begin{tabular}{|c|c|c|c|c|c|c|}
\hline Source laser & $\begin{array}{c}\text { Longueur } \\
\text { d'onde }(\mu \mathrm{m})\end{array}$ & $\begin{array}{c}\text { Mode de } \\
\text { fonctionnement }\end{array}$ & $\begin{array}{l}\text { Puissance } \\
\text { crête }\end{array}$ & $\begin{array}{c}\text { Lentille } \\
\text { focalisatrice }\end{array}$ & $\begin{array}{l}\text { Paramètre } \\
\text { confocal } b \\
\end{array}$ & $\begin{array}{l}\text { Diamètre du } \\
\text { faisceau } W_{0} \\
\end{array}$ \\
\hline $\mathrm{CO}_{2}$ & $\begin{array}{r}10,6 \\
10,3 \\
9,6 \\
9,2\end{array}$ & Déclenché & 1 à $1,5 \mathrm{~kW}$ & $30 \mathrm{~cm}$ & $7 \mathrm{~cm}$ & $330 \mu \mathrm{m}$ \\
\hline $\mathrm{CO}$ & $\begin{array}{l}5,9 \\
5,6 \\
5,1\end{array}$ & Déclenché & $\simeq 0,5 \mathrm{~kW}$ & $30 \mathrm{~cm}$ & $7 \mathrm{~cm}$ & $230 \mu \mathrm{m}$ \\
\hline YAG $: \mathrm{Nd}^{3+}$ & 1,32 & Déclenché & $\simeq 0,3 \mathrm{~kW}$ & $17,5 \mathrm{~cm}$ & $13 \mathrm{~cm}$ & $170 \mu \mathrm{m}$ \\
\hline
\end{tabular}

Caractéristiques principales des sources lasers utilisées

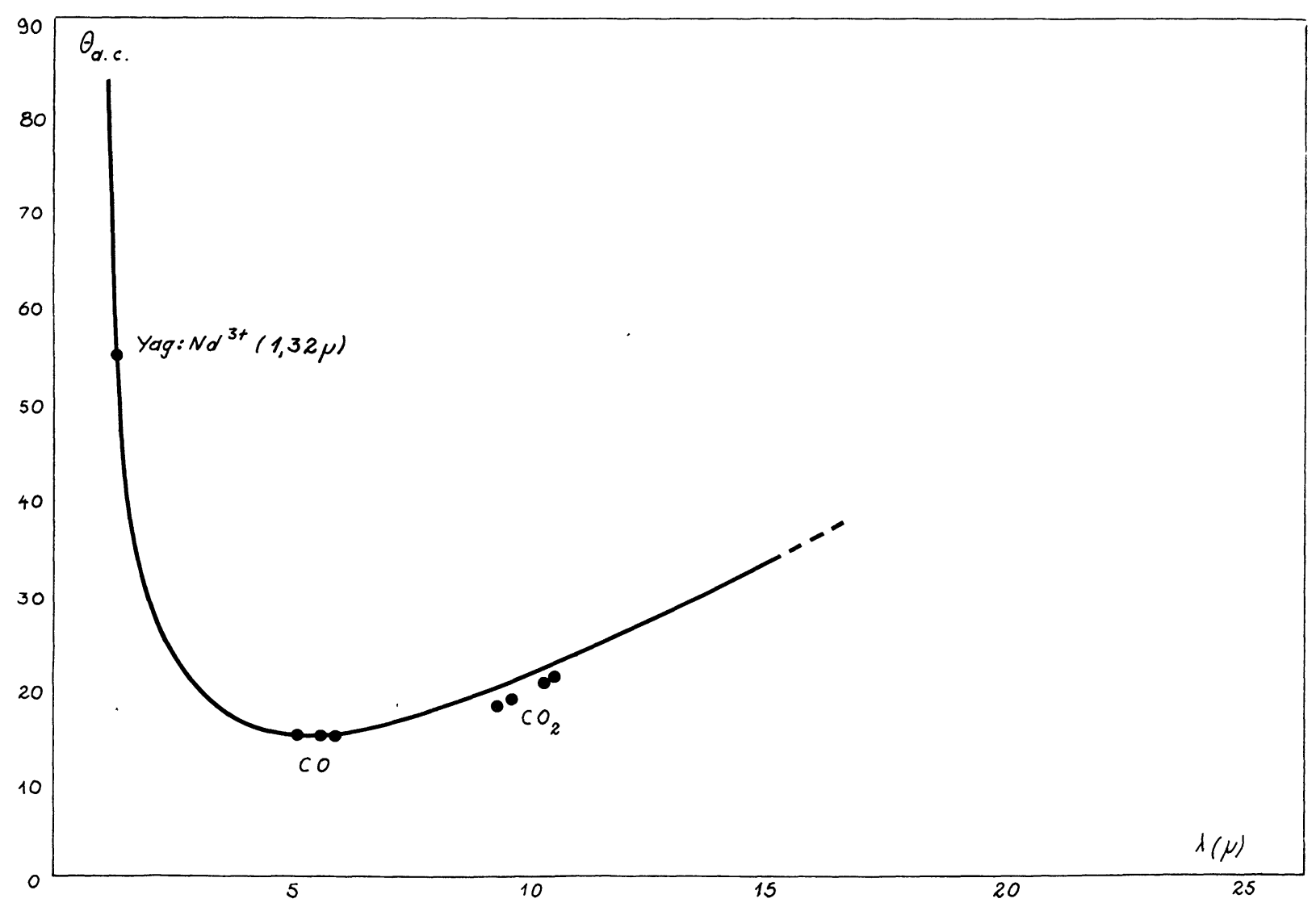

Fig. 1. - Courbes d'accord de phase pour la G. S. H. : $\theta_{\text {a.c. }}(\lambda)$. 
synthétique, taillés dans des lingots de quelques $\mathrm{cm}^{3}$ (Fig. 2), se présentent sous forme de plaquettes d'environ $5 \times 5 \mathrm{~mm}^{2}$ et $1 \mathrm{~mm}$ d'épaisseur. La direction normale aux plaquettes fait approximativement avec l'axe optique l'angle $\theta_{\text {a.c. }}$ fourni par la courbe de la figure 1 à la longueur d'onde considérée. Le matériau, rouge sombre, présente à l'œil nu un certain nombre de

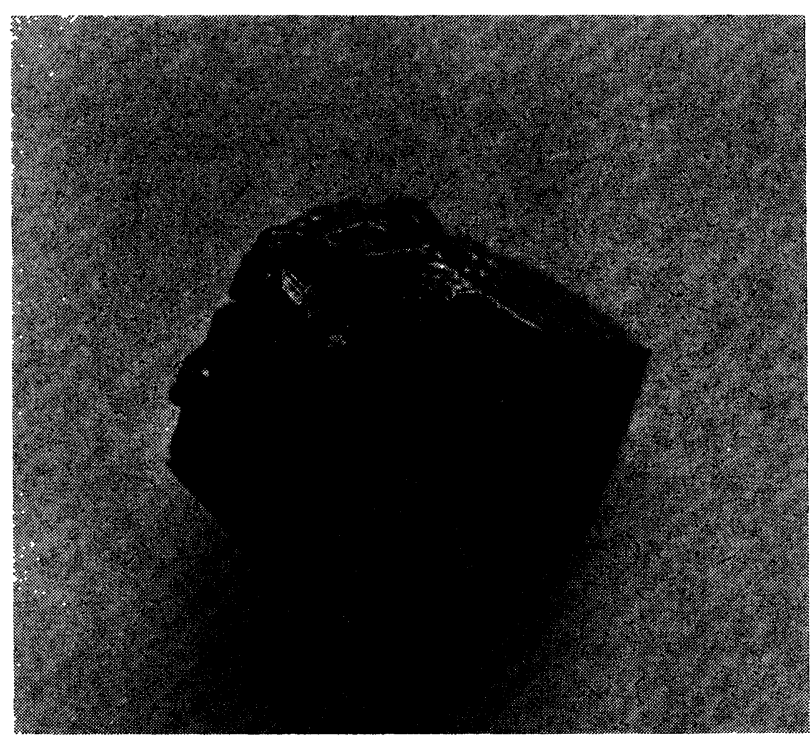

$$
2 \mathrm{~cm}
$$

FIG. 2. - Photographie d'un lingot de cinabre synthétique.

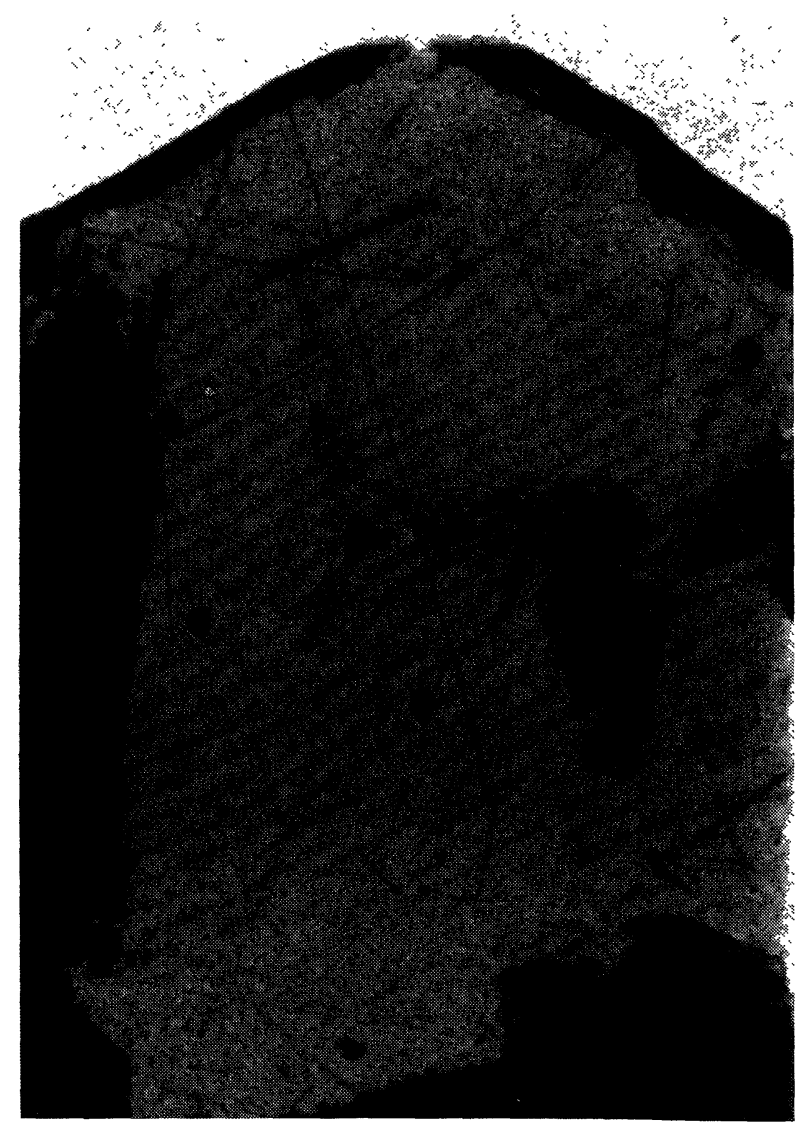

FIG. 3. - Photographie de la plaquette utilisée à $10,6 \mu \mathrm{m}$. défauts tels que trous, rayures et inclusions opaques (Fig. 3).

Après choix de zones cristallines convenables par observation microscopique, nous avons, pour l'ensemble des longueurs d'onde énumérées (Tableau I) obtenu la génération de second harmonique et enregistré les pics d'accord de phase, soit $P_{2 \omega}$ en fonction de l'angle d'incidence $\theta_{\text {ext }}$ (exemple Fig. 4). Ces courbes

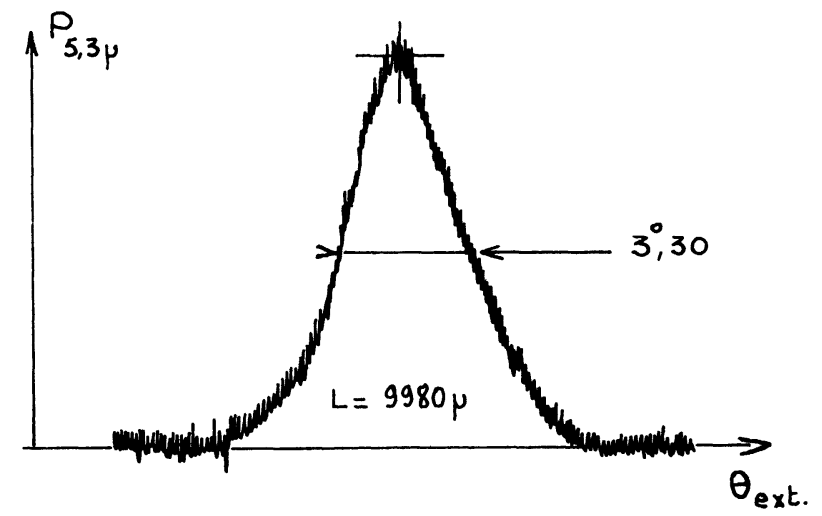

FIG. 4. - Exemple d'un pic d'accord de phase pour la G. S. H., $10,6 \mu \mathrm{m} \rightarrow 5,3 \mu \mathrm{m}$.

permettent de déterminer les angles d'accord de phase. Les valeurs mesurées de ces derniers sont données tableau III et reportées figure 1 . On constate que l'accord est excellent dans le proche I. R. mais qu'un

\section{TABLEAU III}

Angles d'accord de phase mesurés

$\begin{array}{cc}\lambda(\mu \mathrm{m}) & \underline{\theta_{\text {a.c. }}} \\ \overline{10,6} & 20,75^{\circ} \pm 0,75 \\ 10,3 & 20,20^{\circ} \pm 0,75 \\ 9,6 & 18,50^{\circ} \pm 0,75 \\ 9,3 & 17,80^{\circ} \pm 0,75 \\ 5,10 & 14,80^{\circ} \pm 0,75 \\ 5,62 & 14,60^{\circ} \pm 0,75 \\ 5,90 & 14,50^{\circ} \pm 0,75 \\ 1,32 & 54,60^{\circ} \pm 0,50\end{array}$

léger écart se manifeste pour les longueurs d'onde du laser $\mathrm{CO}_{2}$. On remarquera cependant que les valeurs mesurées des indices sont moins nombreuses dans cette région que dans le domaine visible et s'arrêtent précisément dans la zone d'intérêt. La largeur à mi-hauteur $\Delta \theta_{\text {ext }}$ des pics d'accord peut être calculée à partir des valeurs de l'angle de réfraction $\theta_{\text {int }}$, de l'épaisseur $L$ de l'échantillon et de l'angle de double réfraction $\rho$

$$
\Delta \theta_{\mathrm{ext}}=0,443 \frac{\lambda \cos \theta_{\mathrm{int}}}{L \rho} ;
$$

l'accord avec les valeurs expérimentales est satisfaisant (Tableau IV). 


\section{TABLEAU IV}

Largeur à mi-hauteur de deux pics d'accord de phase

$\begin{array}{ccc}\lambda(\mu \mathrm{m}) & \Delta \theta_{\text {ext }} \text { calculé } & \Delta \theta_{\text {ext }} \text { mesuré } \\ 10,6 & 4,7^{\circ} & 3,30^{\circ} \pm 0,25 \\ 1,32 & 0,30^{\circ} & 0,40^{\circ} \pm 0,05\end{array}$

Le coefficient non linéaire du second ordre $d_{11}\left({ }^{1}\right)$, précédemment évalué par J. Jerphagnon sur le cinabre naturel [5], a été mesuré comparativement au coefficient $d^{+}$de la proustite à $10,6 \mu \mathrm{m}$ et du coefficient $d_{36}$ du KDP à 1,32 $\mu \mathrm{m}$. Par ailleurs, nous avons effectué une comparaison directe des coefficients $d_{11}$ de deux échantillons de cinabre, l'un synthétique, l'autre naturel. Compte tenu de l'imperfection des échantillons synthétiques, nous avons, pour ces derniers, choisi le plus intense des nombreux pics d'accord de phase enregistrés en différents points d'un même échantillon et ayant une largeur à mi-hauteur en accord avec la valeur calculée. Pour toutes les configurations expérimentales l'épaisseur des échantillons examinés était faible devant les longueurs d'ouverture $\left(l_{\mathrm{a}}=\sqrt{\pi} W_{\mathrm{o}} / \rho\right)$ et longueur de focalisation $\left(l_{\mathrm{f}}=\pi \mathrm{b} / 2\right)$ caractérisant les faisceaux. Les résultats des mesures ont été analysés selon la méthode utilisée par Kupecek et al. [6] dans le cas de l'accord de phase. Les résultats sont rassemblés dans le tableau $\mathrm{V}$.

La règle empirique de $R$. C. Miller [7] prévoit une dispersion négligeable du tenseur

$$
\Delta_{i j k}=d_{i j k} / \chi_{i i} \chi_{j j} \chi_{k k},
$$

où $d_{i j k}$ est un élément du tenseur de non-linéarité du second ordre et $\chi_{i i}$ un élément du tenseur de susceptibilité linéaire. Cette règle peut être utilisée pour corréler les valeurs d'un coefficient non linéaire à des longueurs

(1) Notons que le résultat direct de la mesure est le coefficient efficace $d_{\text {eff }}=d_{11} \cos ^{2} \theta_{\text {a.c. }}$. d'onde différentes. Son application au cinabre prévoit $d_{11}(1,32 \mu \mathrm{m})=1,4 d_{11}(10,6 \mu \mathrm{m})$. Pour comparer ce résultat à celui de nos mesures, il est nécessaire de faire usage des valeurs absolues des coefficients non linéaires des matériaux étalons. Nous choisissons avec R. Bechman et al. [8] $d_{36}(\mathrm{KDP})=1,5 \times 10^{-9}$ esu et avec D. S. Chemla et al. [9] $d_{+}$(proustite) $=75 \times 10^{-9}$ esu. Ceci conduit à la relation

$$
d_{11}(1,32 \mu \mathrm{m})=(1,8 \pm 0,3) d_{11}(10,6 \mu \mathrm{m}) .
$$

Remarquons alors que l'harmonique à $0,66 \mu \mathrm{m}$, soit $1,88 \mathrm{eV}$, n'est pas très loin de la bande interdite du cinabre, située vers $1,95 \mathrm{eV}$ et qu'une augmentation par résonance du coefficient $d_{11}$ n'est pas exclue. Il est cependant fondamental de noter que toute interprétation de cette dispersion de $d_{11}$ repose sur le raccordement des mesures d'étalons entre le visible et l'infrarouge, problème qui n'a pas encore reçu de solution satisfaisante $(*)$.

Comme nous l'avons signalé, la détermination des angles d'accord et des coefficients non linéaires a été effectuée après un choix optimum de la zone cristalline siège de l'interaction. Ceci signifie que les valeurs issues de nos mesures caractérisent des volumes restreints de longueur égale à l'épaisseur des plaquettes, soit environ $1 \mathrm{~mm}$ et de section $\pi W_{0}^{2}$, soit $0,35 \mathrm{~mm}^{2}$ à $10,6 \mu \mathrm{m}$ et $0,09 \mathrm{~mm}^{2}$ à $1,32 \mu \mathrm{m}$. Dans ces conditions on définit une qualité locale du matériau, caractérisée par un coefficient non linéaire élevé et une faible absorption résiduelle.

3. Qualité d'ensemble du cinabre de synthèse. La possibilité d'utiliser le cinabre de synthèse comme élément d'un dispositif paramétrique optique est liée à l'existence de cristaux de grandes dimensions, possédant sur l'ensemble du volume les qualités requises de coefficient non linéaire élevé et de faible absorption résiduelle. Le matériau doit par ailleurs présenter une rigoureuse homogénéité des indices de réfraction et une parfaite régularité de la structure cristalline. Les

\section{TABLEAU V}

Valeurs relatives $d u$ cofficient $d_{11}$

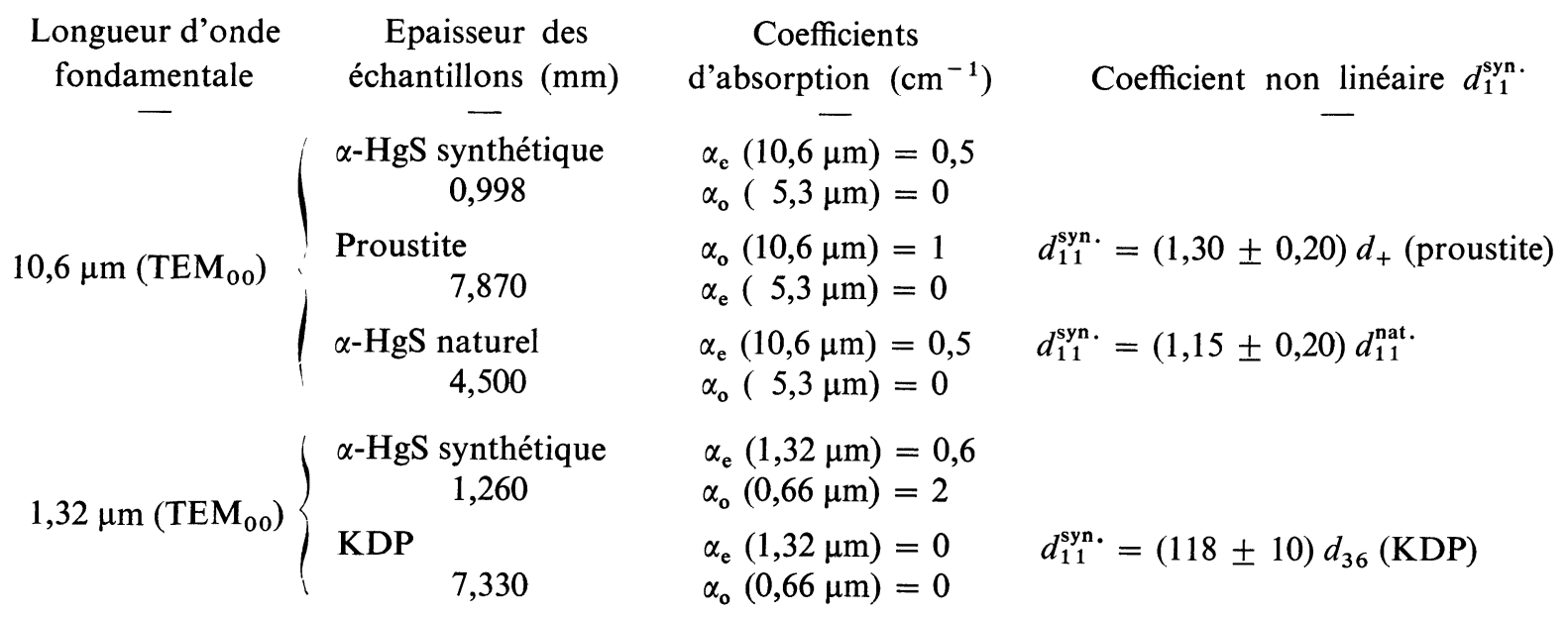


mesures précédentes effectuées dans des régions choisies de faible volume ont montré une bonne qualité locale. Il est aussi nécessaire d'examiner en détail si ces qualités se retrouvent sur la totalité d'un échantillon. L'ensemble des tests a été effectué à l'aide d'une source laser à $10,6 \mu \mathrm{m}$.

3.1 HoMOGÉNÉITÉ DE LA TRANSMISSION. - Le faisceau du laser $\mathrm{CO}_{2}$ est focalisé en un pinceau de $100 \mu \mathrm{m}$ environ de rayon sur le cristal à étudier. Ce dernier approximativement perpendiculaire au faisceau incident est déplacé parallèlement à lui-même et l'on enregistre l'intensité du rayonnement à $10,6 \mu \mathrm{m}$ transmis (Fig. $5 a, 6 a$ ). Les enregistrements confirment l'existence de défauts dont certains n'étaient pas décelables à l'œil nu.

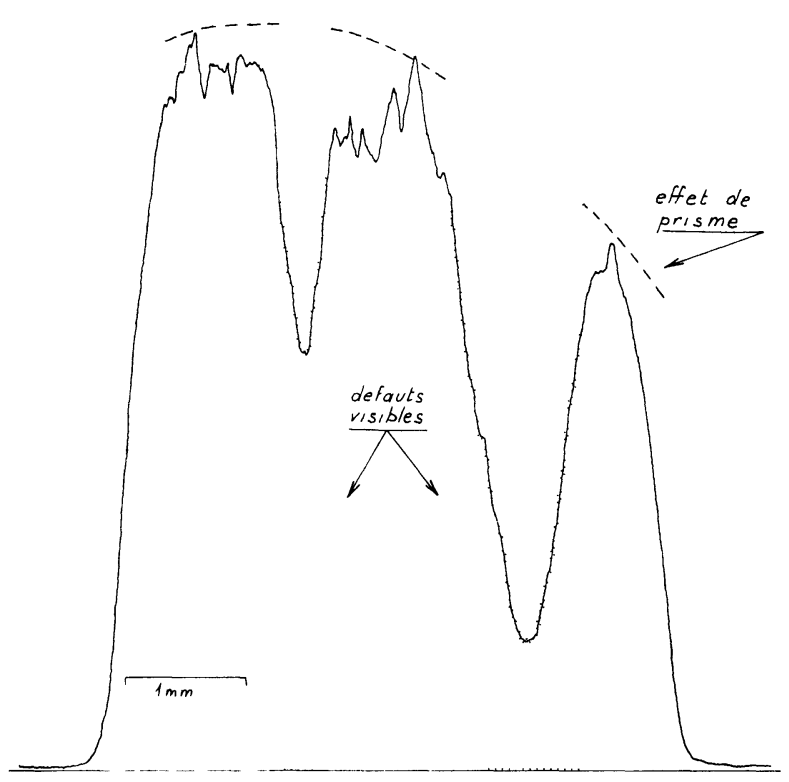

FIG. $5 a$.

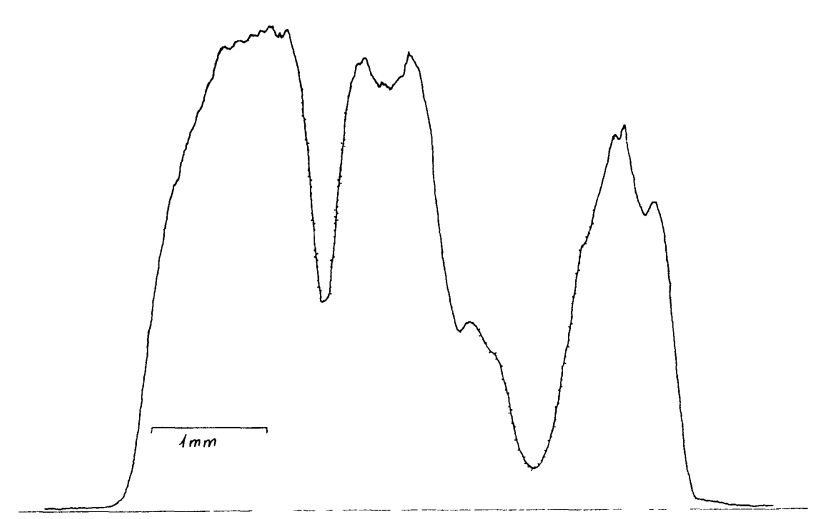

FIG. $5 b$.
De plus, compte tenu de l'indice de réfraction élevé du cinabre, le défaut de parallélisme des lames amène une lente variation de la transmission dont il convient de tenir compte (ligne interrompue, Fig. 5a).

3.2 HoMOGÉNÉITÉ DE LA GÉNÉRATION D'HARMONIQUE A $5,3 \mu \mathrm{m}$. - Nous avons déjà signalé la haute qualité exigée en ce qui concerne la régularité de l'orientation cristalline dans le matériau. Le processus de G. S. H. à l'accord de phase est un test particulièrement sévère. Si l'on considère par exemple la courbe de la figure 4 , on constate qu'une désorientation relative entre le vecteur d'onde et l'axe optique de $35^{\prime}$ d'arc amène une réduction de $50 \%$ du signal harmonique à $5,3 \mu \mathrm{m}$ (rappelons que l'on doit, pour évaluer cette désorientation, utiliser la relation $\sin \theta_{\text {ext }}=n \sin \theta_{\text {int }}$ ).

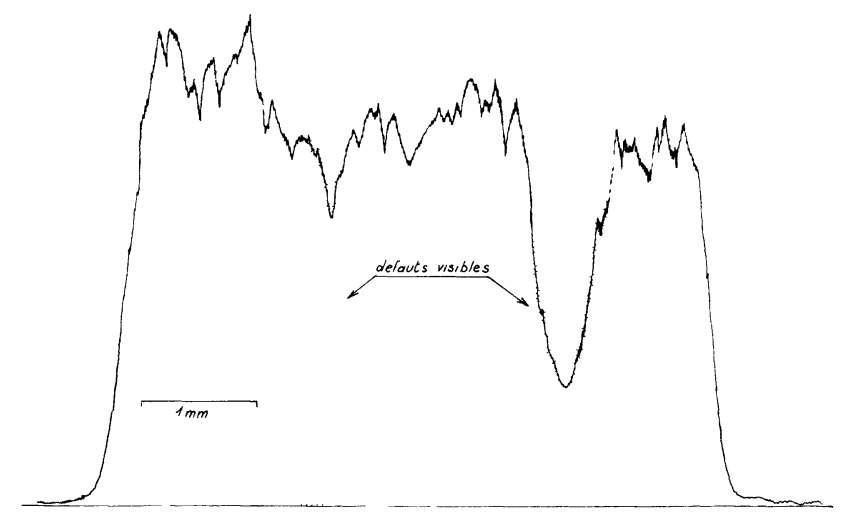

FIG. $6 a$.

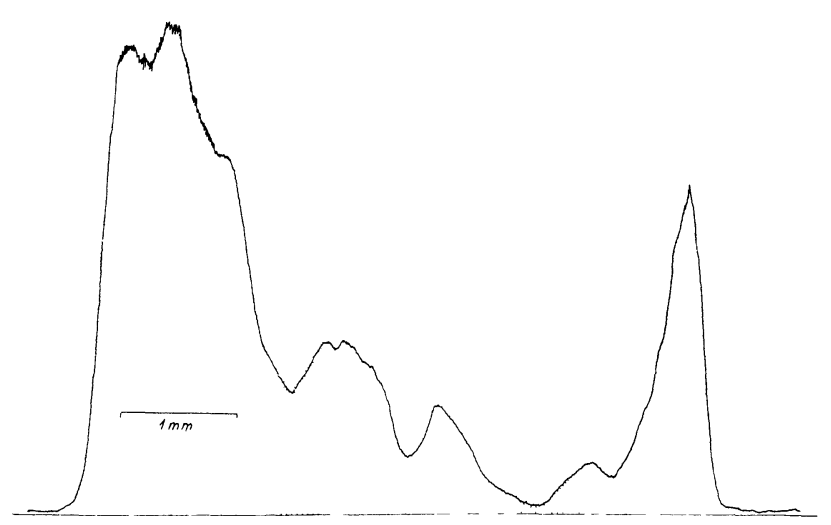

FIG. $6 b$.

FIG. 5 et 6. - $-a$ ) Homogénéité de transmission à $10,6 \mu \mathrm{m}$. b) Homogénéité de la G. S. H. dans les mêmes conditions. 
Ayant réalisé l'accord de phase en un point de l'échantillon, celui-ci est déplacé parallèlement à luimême et l'on enregistre l'intensité du seul signal harmonique. Changeant de filtres, et par un second passage, on enregistre la transmission du signal fondamental dans les mêmes conditions.

Pour certaines zones cristallines (Fig. $5 a, 5 b$ ), l'intensité harmonique varie en fonction de la transmission à 10,6 $\mu \mathrm{m}$. Dans d'autres régions (Fig. $6 a, 6 b$ ) des accidents supplémentaires viennent se superposer à ceux provoqués par les variations de transmission. On peut alors supposer que de légères variations de l'orientation des axes cristallins de l'échantillon sont la cause de ces perturbations. Dans cette hypothèse, en se reportant à la courbe de la figure 4, les écarts maximums d'orientations de l'axe optique peuvent être évalués à une cinquantaine de minutes d'arc.

L'étude aux rayons $X$ confirme cette idée. En effet, les diagrammes de diffraction réalisés en des points différents d'un même cristal montrent une fluctuation de la direction de l'axe optique atteignant $30^{\prime}$ d'arc. Si l'on considère que la pénétration des rayons $X$ est très inférieure à l'épaisseur totale de l'échantillon que le rayonnement à $10,6 \mu \mathrm{m}$ traverse en totalité, les deux résultats sont bien concordants.

3.3 Perturbations éventuelles Causées Par Des MACLES. - Le phénomène général connu sous le nom de macles cristallines a une importance fondamentale lorsque l'on étudie des propriétés physiques liées à l'anisotropie du matériau [10]. Ce phénomène peut apparaître lorsque le groupe des opérations de symétrie (exactes ou approchées) du réseau de Bravais $G_{R}$ contient plus d'éléments que le groupe de symétrie ponctuel du cristal $G_{0}$. L'ensemble des opérations de $G_{0}-G_{\mathrm{R}}$ peut donner lieu à des macles. Dans le cas particulier du cinabre, ces opérations se regroupent en trois types ayant des effets différents sur le système des axes cristallins, et par conséquent sur les éléments non nuls du tenseur cartésien de non-linéarité du second ordre. Les trois types de macles et leurs conséquences sur le coefficient $d_{11}$ du cinabre sont résumés dans le tableau VI. On conçoit alors que lors d'un processus de G. S. H., la présence de macles conduisant à des changements de signe du coefficient $d_{11}$ sera dramatique.

\section{TABLEAU VI}

Effet des opérations de macles sur le coefficient $d_{11}$

$\begin{array}{ccc}\begin{array}{c}\text { Type de } \\ \text { macle }\end{array} & \begin{array}{c}\text { Opérations de } \\ \text { symétrie }(*)\end{array} & \begin{array}{c}\text { Transformation } \\ \text { de } d_{11}\end{array} \\ \overline{\text { I }} & \overline{6^{\prime}} 22^{\prime} & d_{11} \rightarrow-d_{11} \\ \text { II } & \overline{\overline{3}^{\prime}} 2 / \mathrm{m}^{\prime} & d_{11} \rightarrow-d_{11} \\ \text { III } & \overline{6^{\prime}} 2 \mathrm{~m}^{\prime} & d_{11} \rightarrow d_{11}\end{array}$

(*) Les notations sont celles de Curien et al. [12].

L'observation au microscope polarisant des échantillons utilisés montre que ces derniers sont fortement maclés et probablement constitués d'individus diffé- rents intimement imbriqués en volume. Par ailleurs, les valeurs du coefficient $d_{11}$ obtenues après de nombreuses mesures dans différentes zones convenables d'un même échantillon sont toujours apparues concordantes. La conclusion qui s'impose [10] est que les macles présentées sont du type III qui n'ont pas d'influence sur le coefficient $d_{11}$ mesuré.

Par contre, un inconvénient qui semble lié à la forte densité de macles est le faible seuil de dommages optiques présenté par les échantillons de cinabre synthétiques. En effet, une dizaine d'impulsions successives de 4 à $5 \mathrm{MW} / \mathrm{cm}^{2}$ du laser à $\mathrm{CO}_{2}$ font apparaître des cratères dont l'éclat métallique est vraisemblablement dû à la présence, après irradiation, de mercure à l'état libre. A l'inverse, le cinabre naturel, quasiment exempt de macles, n'est pas endommagé par plusieurs milliers d'impulsions de même intensité.

4. Conclusion. - L'état actuel de la cristallogenèse ne permet pas d'envisager dans l'immédiat l'utilisation du cinabre synthétique comme composant d'un dispositif nécessitant un volume important de matériau de qualité, ou possédant un seuil en puissance, tel qu'un oscillateur paramétrique. Par contre, comme convertisseur de fréquence extra-cavité, il peut être fait usage de ses qualités telles que son large domaine de transparence. Nous avons réalisé en particulier, dans un montage déjà utilisé avec du GaSe [11] le mélange additif de fréquences entre les raies d'émission d'un laser à $\mathrm{CO}_{2}$ et d'un laser à $\mathrm{CO}$ déclenchés en synchronisme. L'angle d'accord est voisin de $16^{\circ}$. Cette opération permet d'obtenir plus de mille raies monochromatiques situées entre $3,25 \mu \mathrm{m}$ et $3,85 \mu \mathrm{m}$, de largeur voisine de $70 \mathrm{MHz}$.

Les certitudes étant acquises en ce qui concerne les qualités potentielles du cinabre, son avenir dans des dispositifs optiques raffinés est entièrement tributaire de l'évolution future des méthodes de préparation.

Remerciements. - Nous adressons nos remerciements à Y. Toudic pour la fourniture des lingots de cinabre et à G. Pierson pour la préparation des différents échantillons, ainsi qu'à J. Jerphagon pour l'intérêt qu'il a constamment apporté à ce travail.

(*) Note ajoutée aux épreuves. - Des résultats nouveaux concernant le raccordement visible-infrarouge obtenus à l'Université de Stanford (Californie) par le Professeur R. L. Byer nous ont été communiqués après soumission de notre article. Les nouvelles valeurs recommandées pour les étalons sont :

$$
\text { à } 1,32 \mu \quad d_{36}(\mathrm{KDP})=1,43 \times 10^{-9} \text { esu }
$$$$
\text { à } 10,6 \mu \quad d_{14}(\mathrm{GaAs})=360 \times 10^{-9} \text { esu }
$$

ce qui conduit à $d_{+}$(Proustite) $=84,2 \times 10^{-9}$ esu et à

$$
d_{11}{ }^{\mathrm{HgS}}(1,32 \mu \mathrm{m})=(1,54 \pm, 25) d_{11}{ }^{\mathrm{HgS}}(10,6 \mu \mathrm{m})
$$

en accord avec le résultat prévu par la règle de R. C. Miller. 


\section{Bibliographie}

[1] Toudic, Y., Aumont, R., J. Cryst. Growth 10 (1971) 170.

[2] Kleinman, D. A., Phys. Rev. 126 (1962) 1977.

[3] Bond, W. L., Boyd, G. D., Carter Jr, H. L., J. Appl. Phys. 38 (1967) 4090.

[4] Ayrault, B., Langlois, H., Lecocq-Mayer, M. C., Lefin, F., Phys. Stat. Sol. 17 (1973) 665.

[5] Jerphagnon, J., Batifol, E., Tsoucaris, G., Sourbé, M., C. R. Hebd. Séan. Acad. Sci. B. 265 (1967) 495.

[6] KupeceK, P. J., Schwartz, C. A., Chemla, D. S., IEEE J. Quantum Electron. 10 (1974) 540.
[7] Miller, R. C., Appl. Phys. Lett. 5 (1964) 17.

[8] Bechman, R., KURTz, S. K., Numerical Data and Functional Relationships, Group III, vol. 2, Landolt-Börnstein.

[9] Chemla, D. S., KupeceCK, P. J., Schwartz, C. A., Opt. Commun. 7 (1973) 225.

[10] JERPHAGNON, J., en préparation.

[11] Kupececk, P. J., Batifol, E., Kuhn, A., Opt. Commun. 11 (1974) 291.

[12] Curien, H., Le Corre, Y., Bull. Soc. Fr. Minéral. Cristallogr. 81 (1958) 126. 